\title{
Human Gait Assessment using a 3D Marker-less Multimodal Motion Capture System
}

\author{
Thiago Braga Rodrigues*[0000-0002-2017-4492]1, Debora Pereira Salgado ${ }^{1}$, Ciarán Ó Catháin ${ }^{2}$, Noel O’Connor ${ }^{3}$, \\ Niall Murray ${ }^{1}$
}

\begin{abstract}
Gait analysis is the measurement, processing and systematic interpretation of biomechanical parameters that characterize human locomotion. It supports the identification of movement limitations and development of rehabilitation procedures. Accurate Gait analysis is important in sports analysis, medical field, and rehabilitation. Although Gait analysis is performed in several laboratories in many countries, there are many issues such as: (i) the high cost of precise Motion Capture systems; (ii) the scarcity of qualified personnel to operate them; (iii) expertise required to interpret their results; (iv) space requirements to install and store these systems; as well as difficulties related to the measurement protocols of each system; (vi) limited availability (vii) and the use of markers can be a barrier for some clinical use cases (e.g. patients recovering from orthopedics surgeries). In this work, we present a low cost and more accessible system based on the integration of a Multiple Microsoft Kinect sensors and multiple Shimmer inertial sensors to capture human Gait. The novel multimodal system combines data from inertial and 3D depth cameras and outputs spatiotemporal Gait variables. A comparison of this system with the VICON system (the gold standard in Motion Capture) was performed. Our relatively low-cost marker-less multimodal motion generates a complete 360-degree skeleton view. We compare our system with the VICON via gait spatiotemporal variables: Gait cycle time, stride time, Gait length (distance between two strides), stride length, and velocity. The system was also evaluated with knee and hip joint angles measurement accuracy. The results show high correlation for spatiotemporal variables and joint angles inside the $95 \%$ bootstrap prediction when compared with VICON.
\end{abstract}

\section{Keywords -3D model, Gait analysis, Motion Capture, Multimodal sensors}

\section{INTRODUCTION}

$\mathrm{G}$ AIT is a repetitive sequence of movements of lower limbs that moves the body forward, while simultaneously maintaining stability of the body[1]. During Gait, one limb acts as a movable support, in contact with the ground, while the contralateral limb advances in the air. It is a cyclic movement as limbs invert their roles with each successive step [2]. In general, Gait analysis involves the measurement, processing, and systematic interpretation of biomechanical parameters that characterize human movement. Through Gait analysis, it is possible to identify limitations in movement and provide information to guide rehabilitation and pre-habilitation procedures for orthopedics surgeries. Currently, Gait analysis is performed in biomechanical laboratories. The data from three-dimensional kinematic systems can be obtained through synchronized infrared and high-speed cameras [3]. It is also possible to capture ground reaction force data via force platforms[4]. The combination of joint movement and angles, in addition to spatiotemporal kinematic and individual anthropometric characteristics, can be used to describe Gait. All these variables can be assessed through Motion Capture technologies[5].

Motion Capture (MoCap) is a term used to describe the process of digitalizing motion, transposing it into a digital format using camera-based and wearable systems [6]. There are two main types of optical (based on cameras) MoCap technologies: markerbased MoCap and marker-less-based MoCap, defined based on the need (or not) to use landmark markers to detect position. Moreover, each technology has its pros and cons in Gait evaluation [7]. Both "outside in" MoCap system types (marked-based and marker-less) are not very portable but can be very precise. For example, a powerful marker-based MoCap technologies such as the VICON [8], supports precise, clear, and accurate gait assessment $[9,10]$. MoCap technologies have been used in cinema, game creation, and in health to evaluate performance of athletes or clinical analysis [11]. This technology records the actions and movements of actors with reference markers placed on specific locations of the body, followed by post processing via specialized

\footnotetext{
*Primary corresponding author: Thiago Braga Rodrigues t.brodrigues@ research.ait.ie Debora Pereira Salgado d.psalgado@ research.ait.ie

Dr. Ciarán Ó Catháin ciaranocathain@ ait.ie

Prof. Noel O’Connor noel.oconnor@dcu.ie

Dr. Niall Murray nmurray@ research.ait.ie
}

1 Faculty of Engineering \& Informatics, Athlone Institute of Technology, Dublin Road, Athlone, |Co. Westmeath, Ireland

2 Faculty of Science \& Health, Athlone Institute of Technology, Dublin Road, Athlone, |Co. Westmeath, Ireland

3 Insight Centre for Data Analytics, Dublin City University, Glasnevin, Dublin 9, Dublin, Ireland 
software[8]. The participant performs movements in Gait laboratories with cameras that captures the light reflected by markers. Movements in such systems are a function of the movement of the reflective markers [12]. Despite the fact that this type of system is highly precise and widely applied in various domains, it has some drawbacks: (a) high cost, some marker-based MoCap can cost more than $\$ 150,000.00$ [13]; (b) complex set up (e.g. it takes time and expertise to set up the system and to add markers at subject before the session); (c) occlusion of markers can occur during the movements or unknown experimental artifacts [7]. In case of occlusions, further processing to fill the gaps is required.

Some MoCap technologies do not require the use of reflective markers. Such techniques are less restrictive, thus giving the impression of greater freedom of movement to the user and can overcome the problem of occlusion artifacts. However, complexity lies in the precise representation of the movement when compared with marker-based capture[6]. The operation of the marker-less MoCap is based on the analysis of the silhouette of the image of the actor who is performing the movement in contrast to the background of the image. The marker-less MoCap technique has been widely used in devices that efficiently perform the task of translating human motion into a 3D coordinate point that can be mapped by the computer[14]. In short, it has been proved the potential of marker-less MoCap technologies in clinical studies which require accurate motion knowledge of subject for diagnosing locomotion difficulties and potential improvements in their performance. The Microsoft Kinect (Kinect)[15], first introduced in November 2010, is a marker-less RGB-D MoCap device developed for the Xbox 360 and Xbox One. The most important functions of MS-Kinect are associated with tracking the user's movements against the interface. For such functionality, hardware components such as an infrared light emitter, multiple video cameras, an infrared camera, a vector of microphones, a three-axis accelerometer and a camera tilt motor are used [16]. For instance, validity and accuracy of the Kinect in clinical assessment is strong regarding postural control and standing balance, neurological rehabilitation. Beyond the scope of biomechanics and rehabilitation, such camera-based systems can play an important role in computer vision recognition [17, 18]. It is acknowledgeable that MoCap systems are used in assistance of different languages, sign language, and also identification of items and in improving road safety [19-22].

Although Gait laboratories have become more precise with the development of accurate MoCap devices like VICON system, the cost of equipment is inhibitive for wide scale adoption [23]. A multimodal Gait analysis system that can provide valid data and feedback using inexpensive MoCap devices like the Kinect and inertial sensors would be a welcome alternative to these expensive systems for Gait laboratories. The use of low-cost MoCap such as the multimodal Gait analysis system proposed in this work can be an alternative for motion analysis, orthopedics as in prevention, diagnostics, and rehabilitation of chronic injuries [24].

The aims of this study include, as main contribution the application of a multimodal system in Gait analysis to extract Gait features by combination of inertial and $360^{\circ}$ skeletons. A comparison between our system and the gold standard VICON system in terms of Gait angles and spatiotemporal Gait is performed. In addition, our system overview outlines the designed and developed synchronization modules, and real-time streaming protocol and fusion of accelerometer, gyroscope, and magnetometer data for quaternion representation in $\mathrm{R}^{4}$. The remainder of this paper is structured as follows: discussion of results and comparison across some related work, conclusion which includes future work and potential applications.

\section{RELATED WORK}

First, we review some related Motion Capture works including marker-less systems, inertial based systems, and combined multimodal systems.

\subsection{Marker-less technologies}

Marker-less MoCap technologies have being used in Gait analysis to detect Gait events and also in rehabilitation. Some markerless technologies apply RGB cameras in Gait. In[25], a 2D marker-less Gait analysis was proposed using a single depth camera. Their system had an upright calibration protocol and tracks pelvis and feet segments. They compared their results with a markerbased technology with correlation between 0.82 and 0.99 . Their system contained spatiotemporal variables and stride time error less than 0.02s. The authors in [26] have proposed a Gait analysis system composed by only a single RGB camera. The methodology applied generates a silhouette using particles filtering from a synthetic image. The authors reported that the sample rate was quite low $(20 \mathrm{hz})$ and as a result, they could not get angle changes of more than $5^{\circ}$ between two frames. They reported results for longitudinal displacement of the knee and ankle. Their comparison with the system with VICON system was presented via graphical differences with no calculations. Some movements and joints are occluded due to the limitation of using a single view camera. In[27], a multi Kinect system was developed for Gait assessment. This technique converted skeleton frames from a single Kinect into a feature vector. This work considered "center of mass" (COM) as center of hip, shoulder and spine joints. They also captured stride times, and angular velocities in different phases of stride.. Their system was compared with reference values from Kinect and did not consider any other comparison method with a gold standard system like the VICON system. The results have shown that multiple Kinects can be applied to capture human movement and assess Gait. They have applied the system to capture spatiotemporal Gait variables and found correlation of 0.97 for right stride length, 0.83 for left stride length, and 0.92 for Gait time. Our results have shown correlation of 0.99 for Gait time and 0.95 for right and left stride length. In our work, we strictly compared the multimodal approach (optical mark-less combined with inertial sensors) with the marker-based VICON system.

In [28], the authors proposed a low-cost marker-less MoCap system using a single Microsoft Kinect to obtain 3D joint position 
and extract Gait spatiotemporal features. Their method defined toe contact to mark starting and ending points of a full Gait cycle (full walk) and generated Gait variables. They considered the variables of: speed; step length (distance between two steps); step time; stride length (distance between two strides); and stride time. This study defined what are some of the most important Gait spatiotemporal variables in use today. They compared the system with the 3DMA (3D Motion Analysis) system [29] and concluded that poor detection of anatomic landmarks in lower body caused the system to be inaccurate to get some variables such as step time (diff between Kinect and 3DMA -0.17), and stride time (diff between Kinect and 3DMA -0.20).

The studies above inform that the tracking of a single view MoCap has some limitations: the device cannot distinguish the frontal and back view of an individual, and, because the skeleton is generated by position estimation, errors in the detection can occur for lateral views. In addition, different joints have variations in accuracies e.g. lower joints are less accurate against upper joints[30].

To overcome the limitations outlined above and to have a $360^{\circ}$ of tracking area, increasing the field of view and skeleton detection, numerous authors have proposed the use of multiples Kinects for Gait analysis in their studies. In [31], a multi Kinect system was developed for gait assessment comparing results with VICON. The results have shown that multiple Kinects can be applied to capture human movement and assess Gait. They have applied the system to capture spatiotemporal gait variables and found correlation of 0.97 for right stride length, 0.83 for left stride length, and 0.92 for Gait time. Our results have shown correlation of 0.99 for Gait time and 0.95 for right and left stride length.

Although these authors found out that their system can track motion in one, two or more viewpoints, their system was not capable of tracking full $360^{\circ}$ motion and did not consider the view from the back. The proposed Gait system from this work considers the full $360^{\circ}$ view from users and can capture 3D, spatial and temporal Gait variables.

\subsection{Inertial-based Technologies}

Inertial measurement Units (IMUs) are electronic devices capable of measuring different parameters of a body like acceleration, force, angular rate, and magnetic field around an object. IMUs can provide accurate temporal Gait variables using its internal sensors like accelerometers, gyroscopes, and magnetometers [32]. In [33], the authors used 9DOF IMUs for Gait temporal assessment and concluded that IMUs could be used to assess temporal Gait parameters with high correlation between IMUs and gold standard systems ( 0.97 for Gait time and 0.82 for stride time).

The authors in [34] developed a real-time estimation of temporal Gait variables using 6DOF IMUs and have correlation results of 0.93 for stride time, and 0.97 for velocity against the GAITRite (GR) system [35][34][33][33][33][33][33]. These studies have shown that IMUs can offer a low-cost tool for temporal Gait variables. To have a system that can provide spatial and temporal gait variables at same time, a multimodal system can be a low-cost alternative for the gold standard Gait systems.

Our recent published work has dealt with fusing IMU and multiple depth sensors for Gait angles. We compared results of a single Kinect, fused Kinect, IMU, and combined sensors with Vicon system. We reported principal component analysis of knee and hip angles, which resulted in comparison across individual sensors. The full paper is available at [24].

Considering these works and to the best of the author's knowledge, the novelty of the system proposed here lies in the fact that: (a) no works in the literature have integrated IMUs with a fused Kinect skeleton from multiple Kinect by the combination of 3D points (x, y, z) and quaternion orientation (w, i, j, k) generating in a $360^{\circ}$ view; (b) it presents a real-time wireless synchronization and streaming protocol for multiple IMUs; (c) it supports easy set up and is low cost; (d) it provides 3D and kinematic data with 9 degrees of freedom; (e) it enables fully body reconstruction; (f) it provides accurate join angles; (g) it is marker-less and can be used in any environment. To examine the utility of our system, we compared our system with VICON. In the next section, we introduce our Gait system and discuss the results of system testing. The proposed system framework, signal processing, and experimental protocol is also described. 


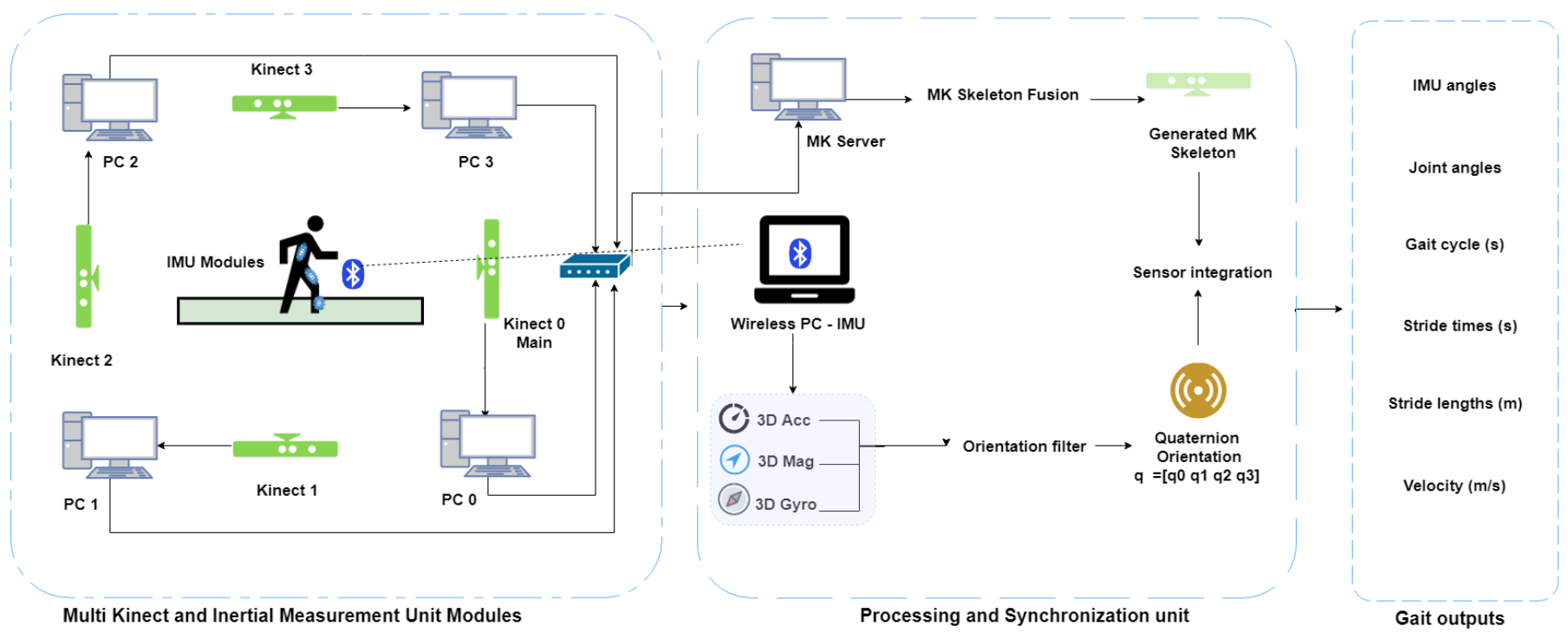

Fig. 1. Gait System diagram containing 4 Kinect skeletons and 4 IMU data as inputs and a fused $360^{\circ}$ skeleton, angles, and Gait spatiotemporal variables as outputs.

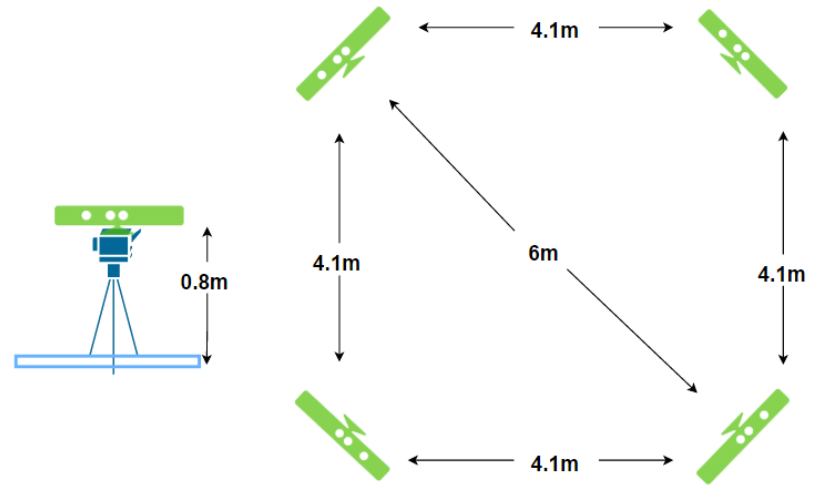

Fig. 2. Kinect arrangement setup. This setup was provided to ensure accurate data capture and synchronization.

\section{PROPOSED HUMAN GAIT ASSESSMENT SYSTEM}

In this section, we give experimental and computational specifications of the Gait system and discuss coordinate transformations, MK skeleton fusion, IMU synchronization and angle evaluation.

\subsection{System setup}

The Gait assessment system captures and combines metadata of 4 MS Kinect cameras, and data from 4 Shimmer IMU. The multimodal architecture is composed by a Multi Kinect module, and an IMU module as per Fig.1. It also contains real-time synchronization protocol and an orientation filter [36] .

\section{A) Multi Kinect Module}

Being aware of the limitations of a single Kinect such as occluded joints and limited area of movement (only front view), a module containing 4 MS Kinects was developed. Each Kinect was powered by its own computer (4 quadcore Intel Core i7, 16GB DDR3 RAM, 3.2Ghz and Graphics Processing Unit running the Kinect for Windows SDK 2.0[37]). The master server communicated with each of the MS Kinects via Transmission Control Protocol (TCP). The master processed the data from each MS Kinect and generates the fused 3D skeleton. Each MS Kinect captures a skeleton from one view perspective and the MultiKinect fusion enables full human body MoCap in 4 views being achieving $360^{\circ}$. The Multi Kinect system contains 3 components: input, processing, and output as per Fig.1. The input component consists of 4 skeletons (one for each Kinect). The processing component is responsible for synchronization, calibration, noise reduction, and skeleton fusion. The output component returns the original 4 skeleton data and a fused $360^{\circ}$ skeleton. For calibration purposes, every two adjacent Kinects were erected perpendicular to each other and all Kinects were kept at same height ( 0.8 meters). The distance between MS Kinects in a square arrangement was 4.1 meters and the diagonal distance was $6 \mathrm{~m}$ as per Fig. 2. This arrangement remained consistent for all experiments. 


\section{Coordinate Transformations}

To use more than one Kinect at same time, the local 3D coordinate system of each Kinect must refer to same 3D global coordinate system. For this experiment, we chose the frontal Kinect $\left(\mathrm{K}_{\mathrm{xyz}}\right)$ as the "master" Kinect and all other Kinects coordinate systems were changes into the $\mathrm{K} 0$ global coordinate system. For this purpose, transformation matrix must be discovered for each Kinect $(\mathrm{K} 1-\mathrm{K} 3)_{\mathrm{xyz}}$ into $\mathrm{K} 0_{\mathrm{xyz}}$ as in (eq. 1). Using the coordinate transformation relationship as per (eq. 3), one Kinect's skeleton coordinate system can be transformed to the second Kinect's skeleton coordinate system by applying a transformation matrix. As a result, the skeleton coordinates in both Kinect skeleton are representing the same coordinate data system. To discover the coordinate transformation matrix, we adopted a closed-form solution using unit quaternions to get a $4 \times 4$ transformation matrix[38]. Considering:

$$
M_{A B}=\left(\begin{array}{cccc}
R_{[0][0]} & R_{[0][1]} & R_{[0][2]} & T x \\
R_{[1][0]} & R_{[1][1]} & R_{[1][2]} & T y \\
R_{[2][0]} & R_{[2][1]} & R_{[2][2]} & T z \\
0 & 0 & 0 & 1
\end{array}\right)
$$

a) $\mathrm{M}_{\mathrm{AB}}-4 \mathrm{x} 4$ transformation matrix to change one local Kinect $\mathrm{A}$ into a global coordinate $\mathrm{B}$.

b) $R[\mathrm{~m}][\mathrm{n}]-$ The $3 \times 3$ rotation matrix

c) $\mathrm{T}$ - The $3 \times 1$ translation vector

To discover the transformation matrix of each Kinect (local coordinate system) into the K0 global coordinate system, we applied (eq. 2).

$$
\begin{aligned}
& B i=s R * A i+T \\
& \left(\begin{array}{l}
x^{\prime} \\
y^{\prime} \\
z^{\prime}
\end{array}\right)=s\left(\begin{array}{lll}
R_{[0][0]} & R_{[0][1]} & R_{[0][2]} \\
R_{[1][0]} & R_{[1][1]} & R_{[1][2]} \\
R_{[2][0]} & R_{[2][1]} & R_{[2][2]}
\end{array}\right) *\left(\begin{array}{l}
x \\
y \\
z
\end{array}\right)+\left(\begin{array}{c}
T x \\
T y \\
T z
\end{array}\right)
\end{aligned}
$$

d) $\mathrm{Bi}-3 \mathrm{xN}$ matrix representing the unit quaternion of the Kinect global 3D point

e) $\mathrm{Ai}-3 \mathrm{xN}$ matrix representing the unit quaternion of the Kinect local 3D point

f) $\mathrm{s}-$ Scale factor if needed (default 1)

To obtain the transformation matrix and calibrate one Kinect skeleton to K0 skeleton, at least four joints must be detected by the two calibrating Kinect at the same time. However, in order to ensure proper calibration, we only assume that captured skeleton data is reliable when the person is standing in front Kinect sensor, and two adjacent Kinects can track all 20 joints at the same time in a trial. To get the most accurate transformation matrix, 120 frames of reliable skeleton data are captured to get the matrix with minimum coordinate difference. The sum of the 20 joints coordinate difference values between calibrated Kinect and KO is calculated as per (eq. 4).

$$
C D V_{i}=\sum_{j=0}^{19}\left(\left[\begin{array}{c}
A_{j} \cdot x \\
A_{j} \cdot y \\
A_{j} \cdot z \\
1
\end{array}\right]-M_{A B} *\left[\begin{array}{c}
B_{j} \cdot x \\
B_{j} \cdot y \\
B_{j} \cdot z \\
1
\end{array}\right]\right),(i=0,119)
$$

g) $\mathrm{CDV}_{\mathrm{i}}-$ The $\mathrm{i}^{\text {th }}$ coordinate difference sum in 120 frames

h) $A_{j}-$ The $j^{\text {th }}$ joint vector of Kinect $A$

i) $B_{j}-$ The $j^{\text {th }}$ joint vector of Kinect $B$

By comparing 120 sums of the coordinate difference values, the transformation matrix with minimum coordinate difference sum is chosen. Noted that because sampling frequency of the multi Kinect system is around $35 \mathrm{~Hz}$, an oversampling to $40 \mathrm{~Hz}$ was employed to synchronize the multimodal system.

\section{The Multi Kinect Skeleton Fusion}

Each Kinect skeleton contains 20 3D joints. The Kinect SDK provides the joint tracking state of every joint and it is important in our study to determine each transformation matrix. This property has three values: "Tracked", "Inferred", and "NotTracked". "Tracked" indicates that the joint is detected by the depth frame. "Inferred" indicates the joint is not being captured by the depth frame but there is a calculation to determine joint. "NotTracked" indicates that the joint position is indeterminable. This led us to 
calculate the skeleton confidence (SC) when generating transformation matrixes as in (5). This property is used to filter unreliable data (Skeletons with many "Inferred" and "NotTracked" joints) from Multi Kinects.

$$
S C_{k}=\sum_{j=0}^{19} J S_{j},(k=0, \ldots, 3 ; j=0, \ldots, 19)
$$

a) $\mathrm{SC}_{\mathrm{k}}$ - The skeleton confidence from the $\mathrm{k}^{\text {th }}$ Kinect

b) $\mathrm{JS}_{\mathrm{j}}-$ The $\mathrm{j}^{\text {th }}$ joint tracking state (1 if "Tracked", else 0 )

A single Kinect is ideal to track a user from a frontal side. Hence, for the back-side detection, the Kinect SDK still captures the user as a frontal view, capturing a noncurated skeleton. Based on the SC, we reduce 2 points for the SC calculation if it is a "back" Kinect (K2) as in (eq. 6).

$$
S C_{k}=\left\{\begin{array}{l}
S C_{k}-2, \text { if } k^{\text {th }}=K 2 \text { "back" } \\
S C_{k}, \\
\text { else }
\end{array},(k=0, \ldots, 3)\right.
$$

Due to this limitation, not all Kinects can track a reliable skeleton of the user and the most reliable skeleton is selected as main skeleton. For being the "main" Kinect, K0 has the highest priority, followed by K1, K3, and K2.

Each 2 adjacent Kinects are fused to generate a fused skeleton. The joint weight of each joint of two Kinects are then calculated. To do calculate joint weights, each skeleton confidence and tracking states of both skeletons are combined as in (eq. 7) and based on the joint weight, smother fused skeletons are generated as per (eq. 8). The final fused skeleton is composed of all joints of the smoother skeletons.

$$
J W_{k j}=\left\{\begin{array}{cr}
S C_{k}, & \text { if } \mathrm{JS}_{j}=\text { "Tracked" } \\
S C_{k} / 2, & \text { else }
\end{array}\right.
$$

c) $\mathrm{SC}_{\mathrm{k}}$ - The skeleton confidence from the $\mathrm{k}^{\text {th }}$ Kinect

d) $\mathrm{JS}_{\mathrm{j}}-$ The $\mathrm{j}^{\text {th }}$ joint tracking state (1 if "Tracked", else 0 )

$$
\overrightarrow{S S}_{j}=\frac{J W_{1 j} \cdot \overrightarrow{T A S}_{1 j}+J W_{2 j} \cdot \overrightarrow{T A S}_{2 j}}{J W_{1 j}+J W_{2 j}}
$$

e) $\overrightarrow{S j}_{j}-$ The $j^{\text {th }}$ smoothed joint coordinate vector

f) $\mathrm{JW}_{1}$ and $\mathrm{JW}_{2}-$ The $\mathrm{j}^{\text {th }}$ joint weights of the two adjacent Kinects

g) $\overrightarrow{\mathrm{TAS}}_{1 \mathrm{j}}$ and $\overrightarrow{\mathrm{TAS}}_{2 \mathrm{j}}-$ The j $\mathrm{j}^{\text {th }}$ joint's coordinate vectors of the two adjacent Kinects

\section{B) Inertial Measurement Unit Module and Synchronization}

The IMU module in our work contains 4 Shimmer IMU sensors[39]. Data from these 4 sensors was captured using Bluetooth following the capture protocol we developed. A multi-function MATLAB script was developed to perform the following capture protocol:

- Sampling frequency of all sensors was defined to be $40 \mathrm{~Hz}$ to generate the quaternion and avoid sensor drifting.

- Internal configuration of each IMU to 9DOF;

- Synchronization between the sensors;

- Enabling start/stop of the IMU system data capture.

More specifically in terms of internal configuration of each IMU, 10 streams of data were captured: (i) 3D acceleration from accelerometer $\left(\mathrm{Acc}_{\mathrm{xyz}}\right)$; (ii) 3D angular velocity from gyroscope $\left(\mathrm{Gyro}_{\mathrm{xyz}}\right)$; (iii) 3D magnetic field from a magnetometer $\left(\mathrm{Mag}_{\mathrm{xyz}}\right)$, and (iv) a timestamp. As discussed later in this section, the $\mathrm{Acc}_{\mathrm{xyz}}, \mathrm{Gyro}_{\mathrm{xyz}}, \mathrm{Mag}_{\mathrm{xyz}}$, were fused to provide quaternion representation.

The Shimmer IMU has enough internal memory to store sessions but each of the sensors has its own time clock. Hence, synchronization of all 4 IMU during data capture is required. An algorithm was designed and implemented to achieve synchronization. Pseudo code for this algorithm is provided in Algorithm 1. The function provided in the algorithm can promote real-time wireless synchronization and streaming protocol for multiple IMUs. This protocol fuses, in real-time, accelerometer, gyroscope, and magnetometer 3D data and generates the quaternion orientation. The data is synchronized with the computer CPU clock ensuring no data is lost. 


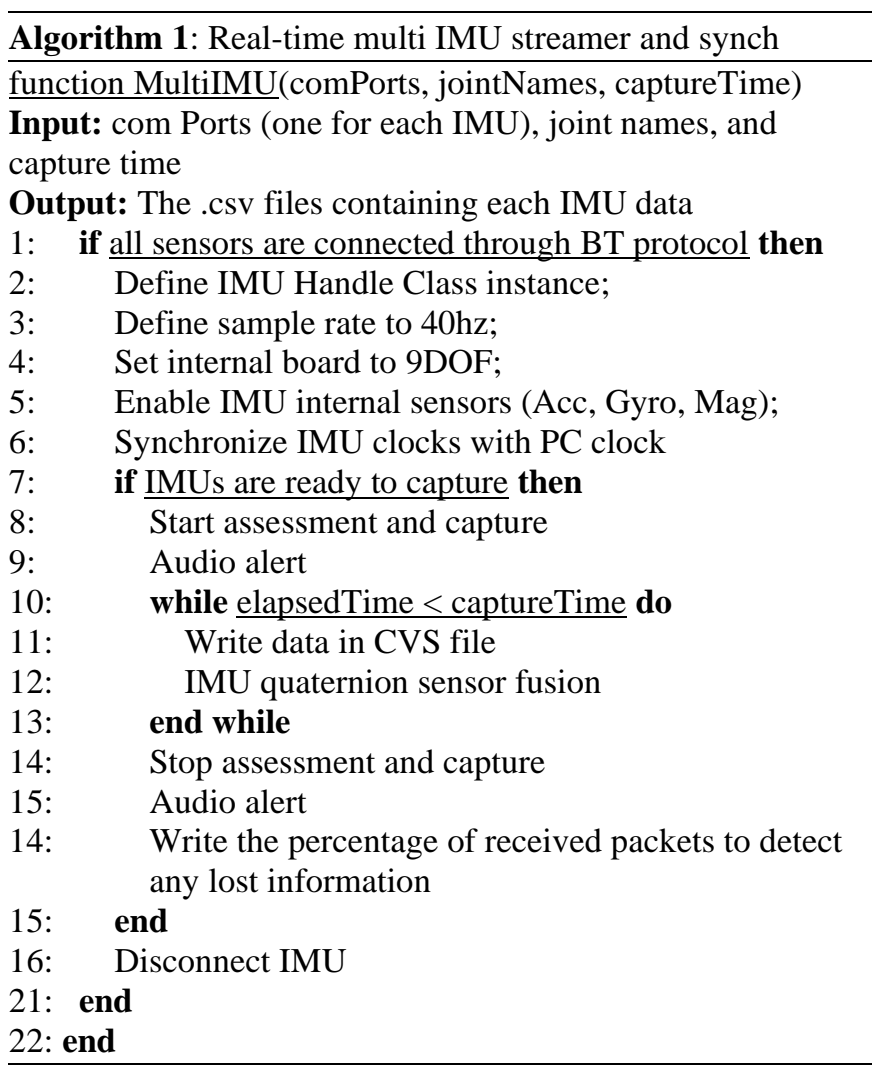

\section{Angle Evaluation}

To represent orientation of a rigid body or frame coordinates in 3D space, a quaternion representation was employed. This complex number representation can define any spatial rotation around a fixed point or coordinate system. A quaternion $q=$

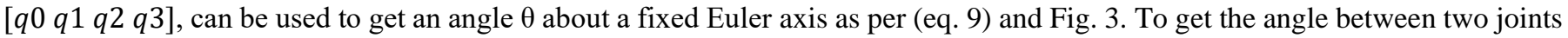
with Shimmer, quaternion matrixes were obtained by the fusion of the 3 Shimmer internal modules ( Acc $_{\mathrm{xyz}}, \mathrm{Gyro}_{\mathrm{xyz}}, \mathrm{Mag}_{\mathrm{xyz}}$ ) $\mathrm{using}$ a Madgwick-based orientation filter[36].

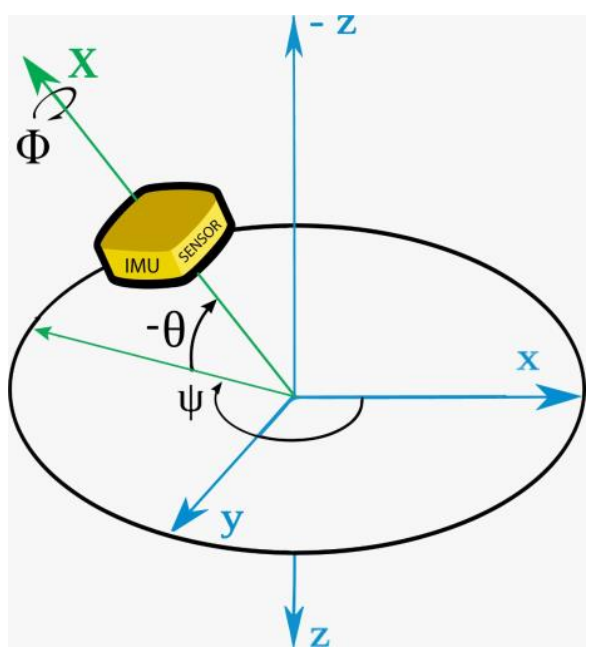

Fig. 3. Rotation angles from IMU.

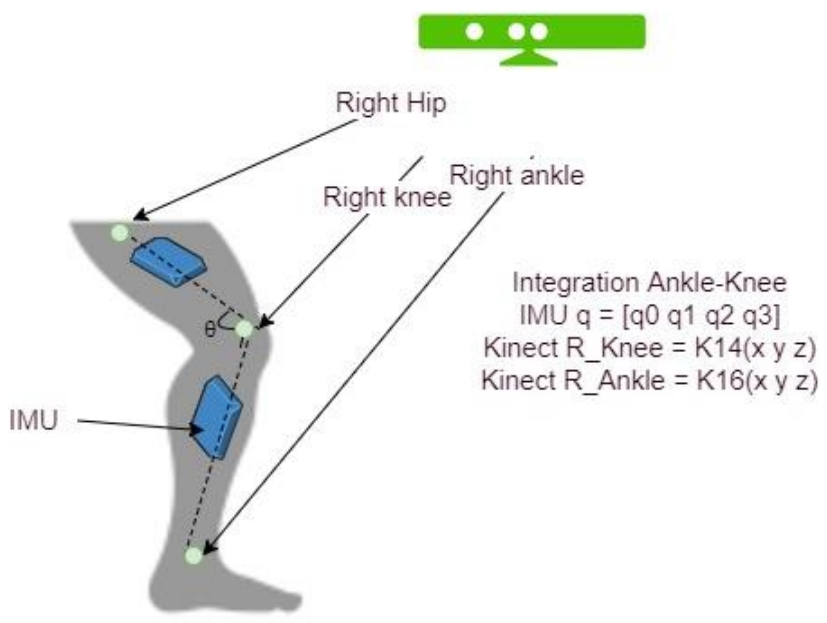

Fig. 4. Quaternion integration. 3D points from Kinect(xyz) are combined with quaternion $\mathrm{q}=[\mathrm{q} 0 \mathrm{q} 1 \mathrm{q} 2 \mathrm{q} 3]$.

The quaternion generated by the function can represent spatial rotation of each shimmer and represents angle in each axis. Having each Euler angle, it is then possible to reference one Shimmer to another and get the angle between two sensors. The angle between two IMU was used as part of the walking evaluation during experiments. The integration of the multi Kinect skeleton and IMU Modules was achieved by combining unit quaternions from 2 Kinect joints Kxyz (for each) and quaternions from the IMU located in the mid-point of those 2 joints $\mathrm{q}=[\mathrm{q} 0 \mathrm{q} 1 \mathrm{q} 2 \mathrm{q} 3]$ (for each) by rotating the quaternion $\mathrm{q}$ around vector $\mathrm{v}$ directing the two Kinect joints $(\mathrm{v}=\mathrm{K} 1-\mathrm{K} 2)$ as per eq. 10 and Fig. 4. The fusion can output projected angles in frontal, lateral and sagittal planes by converting the current rotated quaternion of each IMU to direction cosine matrixes and apply to the Euler angle as per 


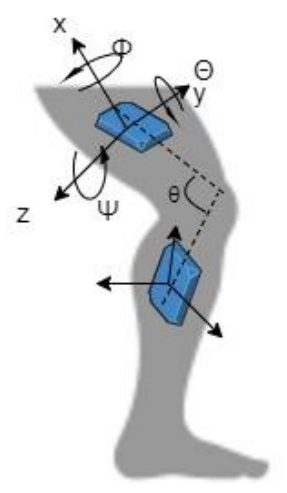

Fig. 5. IMU sensor placement and Euler rotation angle. Each IMU sensor has its own coordinate system and was calibrated before trials to ensure they are in the same coordinate system.

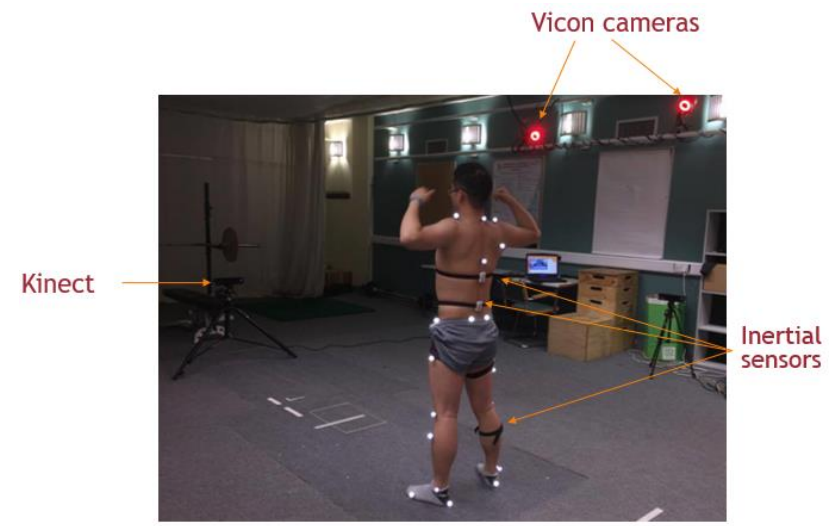

Fig. 6. Static trials. These trials calibrated all streams of data: Vicon and Gait system. Raw data from each sensor was also saved for all trials.

eq 11. The projection angle of any IMU is achieved by trigonometry of the right-angle triangle of IMUxyz of the directional vector from Kinect on the desired plane.

$$
\begin{aligned}
& {\left[\begin{array}{c}
\phi \\
\theta \\
\Psi
\end{array}\right]=\left[\begin{array}{c}
\arctan \frac{2\left(q_{0} q_{1}+q_{2} q_{3}\right)}{1-2\left(q_{1}^{2}+q_{2}^{2}\right)} \\
\arcsin \left(2\left(q_{0} q_{2}-q_{3} q_{1}\right)\right) \\
\arctan \frac{2\left(q_{0} q_{3}+q_{1} q_{2}\right)}{1-2\left(q_{2}^{2}+q_{3}^{2}\right)}
\end{array}\right](9)} \\
& \phi \text { - rotation about the } x \text { axis } \\
& \theta \text { - rotation about the } y \text { axis } \\
& \Psi \text { - rotation about the } z \text { axis } \\
& D C M=\left[\begin{array}{ccc}
\left(q_{0}^{2}+q_{1}^{2}-q_{2}^{2}-q_{3}^{2}\right) & 2\left(q_{1} q_{2}+q_{0} q_{3}\right) & 2\left(q_{1} q_{3}-q_{0} q_{2}\right) \\
2\left(q_{1} q_{2}-q_{0} q_{3}\right) & \left(q_{0}^{2}-q_{1}^{2}+q_{2}^{2}-q_{3}^{2}\right) & 2\left(q_{2} q_{3}+q_{0} q_{1}\right) \\
2\left(q_{1} q_{3}+q_{0} q_{2}\right) & 2\left(q_{2} q_{3}-q_{0} q_{1}\right) & \left(q_{0}^{2}-q_{1}^{2}-q_{2}^{2}+q_{3}^{2}\right)
\end{array}\right] \\
& {\left[\begin{array}{l}
v_{1}^{\prime} \\
v_{2}^{\prime} \\
v_{3}^{\prime}
\end{array}\right]=[D C M]\left[\begin{array}{l}
v_{1} \\
v_{2} \\
v_{3}
\end{array}\right](11)}
\end{aligned}
$$

\section{EXPERIMENTS}

\subsection{Protocol}

The experiments with the Gait system adhere to the approach taken in numerous related works in the literature [40-42] and the protocol employed had the following stages:

- Participants recruitment.

- Information and consent forms.

- Joint measurements.

- $\quad$ Plug-in Gait marker placement[43].

- 8 to 12 Trials per participant (including 1 trial for calibration).

For this study, 8 healthy participants completed 12 trials each, providing a total of 96 individual datasets. For each trial, the participant was asked to jump on a force plate, walk towards the direction of the first Kinect K0 and finish with a second jump. During each trial, motion was captured using our Gait system and VICON simultaneously, hence allowing direct comparison between the two systems. 
Before the experiment, an information sheet was given to each participant to explain the experiment, purposes of the project, and data confidentiality. The participant was also required to sign a consent form. As part of the set-up stage of the participant, reflective markers were placed on the body following the Plug-In-Gait methodology from VICON[43]. Each marker placement and measurement took approximately 45 minutes. After mark placement, joint measurements were taken on the following body segments: arms, legs, height, hip, and shoulders. Finally, the 4 Shimmer IMUs were attached to the participant's body located at mid-points of chest, sacrum, thigh, and tibia (Fig. 5). Before the data collection participants, static trials were performed to calibrate the Multi Kinect system, the IMUs, and VICON systems (Fig. 6). A single trial was achieved when the participant completed a full gait cycle $0-100 \%$. The three streams of data (Kinects, Shimmer, and VICON) were normalized and synchronized for data processing.

\section{RESUltS AND DISCUSSION}

Because the analysis was done with different inputs, the dataset for processing consisted of 3 main frames of information: MultiKinect system, Shimmers, and VICON. The Multi Kinect dataset of 5 skeletons (4 single Kinect Skeletons and 1 fused Kinect Skeleton) was stored in a .csv file. Each skeleton was composed of 20 joint points. Data from Shimmer IMU was stored in a matrix format as described on section III C. The VICON dataset, like Kinect, was in 3D position format, and was captured (VICON $\mathrm{xyz}_{\text {) }}$ on a per reflective marker basis. During each trial, some of the markers were occluded (a known problem with VICON). Hence, post the test, each VICON trial needed to be processed separately, frame per frame, ensure all gaps were filled using spline fill and pattern fill gap filling operations[44].

To compare VICON and each Kinect three tasks needed to be completed: (1) select Kinect joints that can be related to VICON reflective markers (e.g. same body segment; right arm, right leg, hip); (2) change VICON local coordinate system into Kinect global coordinate system following the same closed solution for unit quaternions as per[38]; (3) synchronize both systems with an

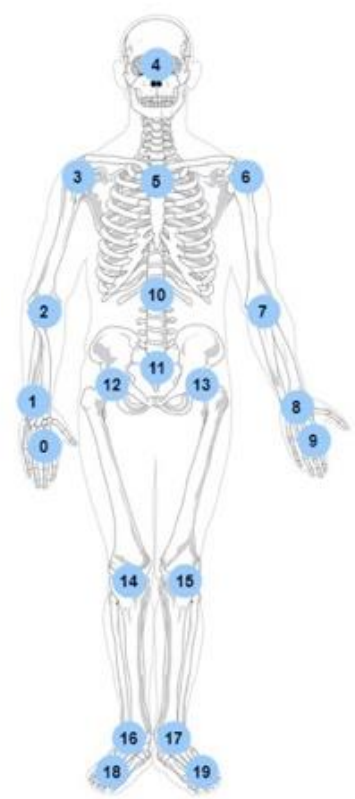

Fig. 7. (a) Kinect joint index, (b) VICON Plug-in-Gait marker placement. Each Kinect joint was related to a VICON marker.

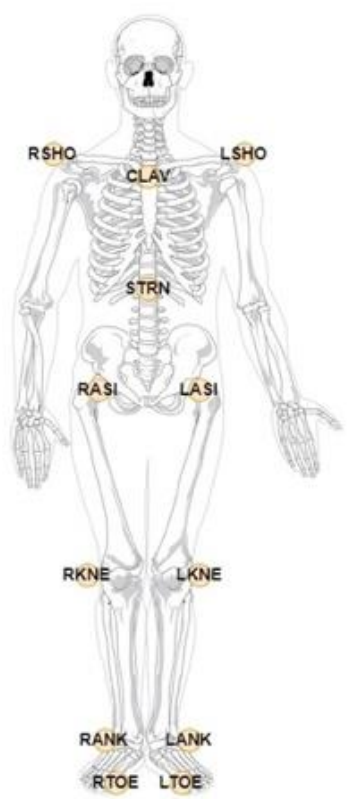

Fig. 8. 3D plot of VICON and Multi Kinect points. Each VICON point is captured by an external marker onto skin. The Kinect joint is inferred by the real 3D anatomic position.

external event. For (3), as outlined above, a jump in a force plate was used and as such each event captured by the force plate generated a trigger for the system.

As per Fig. 7, 12 joints were selected via the Plug-in-Gait and were compared with respective Kinect joints. These were: right shoulder (3_RSHO), shoulder centre (5_CLAV), left shoulder (6_LSHO), spine (10_STRN), right hip (12_RASI), left hip (13_LASI), right knee (14_RKNE), left knee (15_LKNE), right ankle (16_RANK), left ankle (17_LANK), right foot (18_RTOE), and left foot (19_LTOE); all of which are important for GAIT variable extraction and analysis. Each VICON marker is attached onto skin whist Kinect joints are inferred in the anatomic position of each joint as per Fig. 8.

\subsection{Gait Prediction and Confidence Bands}

Hip and knee angles were evaluated as these joints are weight-bearing joints and most susceptible to require bone surgical interventions[45-47]. To evaluate angles from all data sets, two approaches were used: first, having three distinct points in space and second: from the quaternion function and Euler angles from two IMU. The angle between 3 points is equivalent of the angle between two vectors defined by same 3 points. 


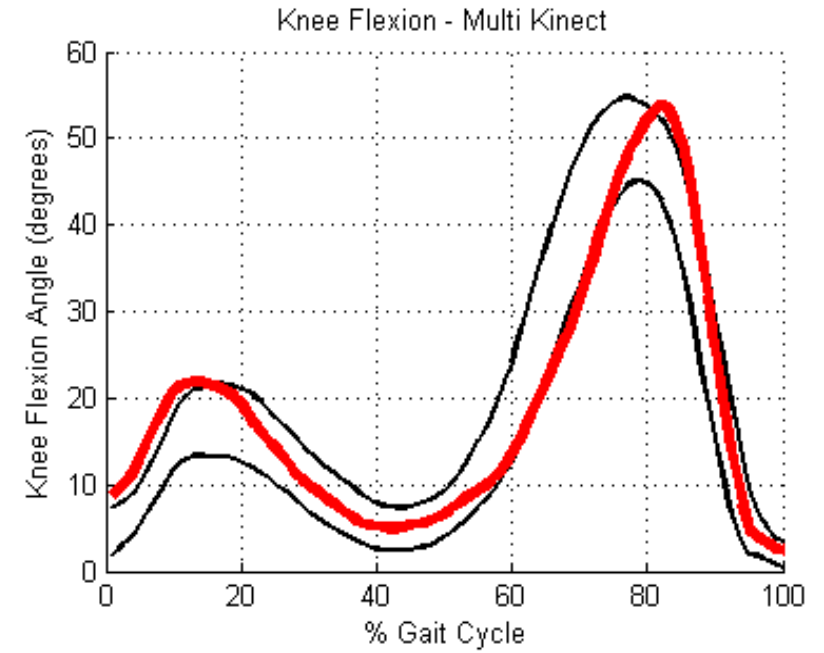

Fig. 9. Knee flexion angles from Multi Kinect module (red) and confidence bands of $95 \%$.

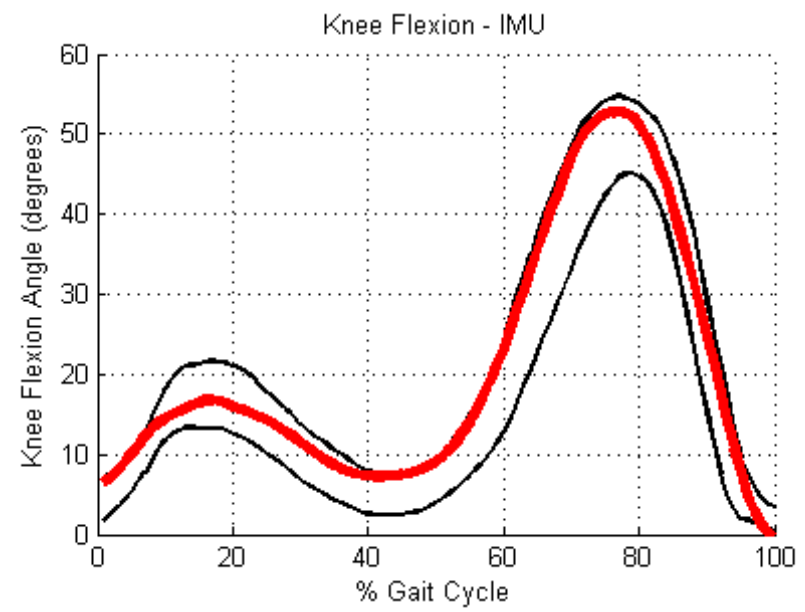

Fig. 10. Knee flexion angles from IMU module (red) and confidence bands of $95 \%$.

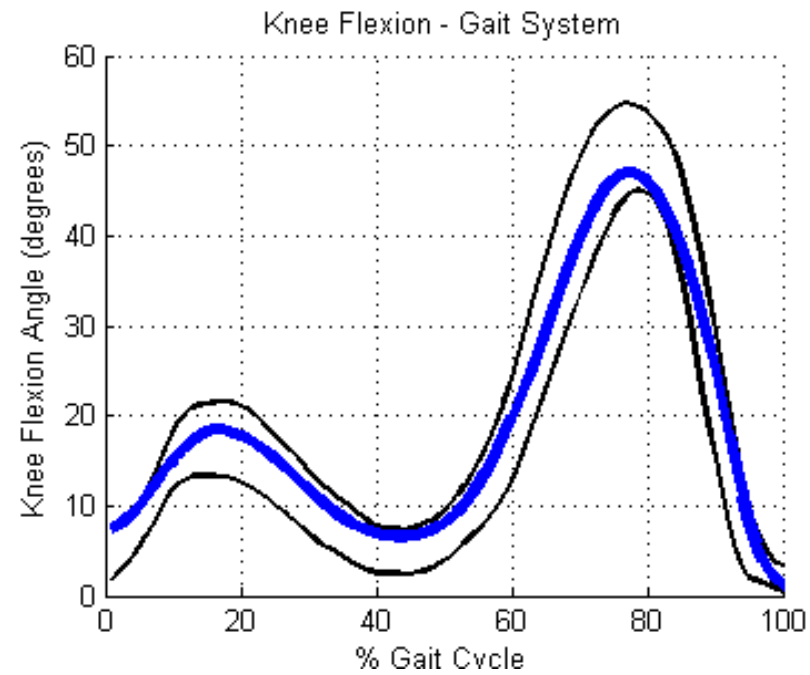

Fig. 11. Knee flexion angles from Gait system (blue) and confidence band of $95 \%$.

Gait analysis often utilizes continuous curves to evaluate a full Gait cycle. The cycle happens when the participant steps on the ground (heel strike), removes the heel stepping with other foot (initial swing), and step on the ground with the same foot in heel strike (terminal swing)[48]. Gait angle data is not composed of single points and cannot be statistically evaluated using common statistical analysis[49]. When dealing with single observations of data, prediction intervals are made for a probability interval. For continuous data, the analogous prediction contains a new prediction every time a new curve is added from population[50]. This method is called bootstrap and can give prediction intervals for Gait curves at any confidence interval[51]. 
TABLE I

SPATIOTEMPORAL RESULTS - VICON VS. GAIT SYSTEM

\begin{tabular}{lllccc}
\hline \hline Variable & Group & Mean & Sd & Sig (2-tailed) & $\begin{array}{c}\text { Pearson's } \\
\text { Correlation } \\
\text { (r) }\end{array}$ \\
\hline Gait Cycle (s) & VICON & 1.308 & 0.037 & 0.714 & 0.996 \\
& Gait System & 1.300 & 0.036 & & \\
Right Stride (s) & VICON & 0.709 & 0.022 & 0.390 & 0.956 \\
& Gait System & 0.700 & 0.014 & & \\
Left Stride (s) & VICON & 0.598 & 0.025 & 0.901 & 0.908 \\
& Gait System & 0.600 & 0.029 & & \\
Gait length (m) & VICON & 0.958 & 0.152 & 0.840 & 0.886 \\
& Gait System & 0.941 & 0.160 & & \\
Stride length (m) & VICON & 0.585 & 0.046 & 0.087 & 0.950 \\
& Gait System & 0.535 & 0.053 & & 0.919 \\
Velocity (m/s) & VICON & 0.734 & 0.127 & 0.892 & \\
& Gait System & 0.724 & 0.130 & & \\
\hline \hline
\end{tabular}

This table compares VICON with the Gait System. The results from the sigma value explains that there is no significant difference between two groups.

Spatiotemporal units are defined; $\mathrm{s}=$ seconds, $\mathrm{m}=$ meters, $\mathrm{m} / \mathrm{s}=$ meters per second of Gait cycle.

Fig. 9, Fig. 10, and Fig. 11 present 95\% bootstrap prediction band of VICON compared with Multi Kinect, IMU, and Gait system. The graphs in red represent the Multi Kinect and IMU outputs whereas the blue represents the Gait System representation. The flexion angles are defined in full Gait cycle. Fig. 12, Fig. 13, and Fig. 14 show the comparison for hip flexion angles for 95\% bootstrap prediction band. The area between two black curves represent the VICON prediction band. Any curve outside this area does not fit the VICON curve, hence it cannot be used to evaluate angles. From the figures presented, the $\mathrm{x}$ axis shows the percentages of Gait from 0 to $100 \%$. The initial phase $0 \%$ happens when the heel contacts the ground and $100 \%$ when the same heel contacts the ground. The y axis represents flexion angles of hip and knee.

For the Knee and Hip flexion curves from Multi Kinect module (Fig. 9 and Fig. 12), since there is a processing time in generating a fused skeleton, filtering noise, and calibrating coordinates, there is also a delay in outputting of Gait graphs and might be an issue where applications demand a precise timing information. The use of the MSc Kinect in applications at higher speed (e.g. Spiriting, fast movements, sports mechanics) is discouraged. The prediction band informs that for initial knee flexion angles $\left(0^{\circ}-20^{\circ}\right)$ and maximum flexion $\left(60^{\circ}-80^{\circ}\right.$ degrees), the MK module was outside the prediction band and could not represent the VICON curve for all degrees. The hip flexion curve for the $60^{\circ}-80^{\circ}$ angles is also outside the prediction band of VICON.

For the Knee and Hip flexion curves from IMU module (Fig. 10 and Fig. 13), there is no delay in outputting Gait graphs. The IMUs can provide information at higher sample rates when compared with Kinect. Initial flexion angles from knee and hip are outside prediction band. The IMU module can represent Gait graphs of hip and knee. However, they cannot represent 3D points and could not be used in applications where 3D and body representation are essential.

The proposed Gait system by the combination of MK and IMU could provide knee and hip angles from Gait as per Fig. 11 and Fig. 14. From these graphs, we can see visual representation of the Gait cycle graphs of the Gait System. The current analysis examined knee and hip angles as these are the main weight bearing joints related to human Gait. Based on the 95\% bootstrap prediction, our Gait system is capable of replicating angles in the sagittal plane of knee and hip and kept the output within the prediction bands. Different analysis of Gait angles across different modules of the system was published in our previous work [24]. Spatiotemporal analysis was also provided for a complete human Gait analysis.

\subsection{Spatiotemporal results}

To evaluate the Gait system, in addition to Gait graphs, it is also important to evaluate spatial variables like Gait and stride length, and temporal variables such as Gait cycle time, stride times, and velocity. For those variables, we have performed an analysis of spatiotemporal variables of walk. All variables of Gait were also compared with the VICON system.

Table I shows results for spatiotemporal Gait variables. It includes statistic results for an independent $t$-test. Assuming equal variances, we have found that both groups VICON and Gait system are comparable, thus the system outputs similar values. We found out lower accuracy of the MS Kinect for some lower joints like ankles and toes. This could interfere on the capability of the system to provide Gait length $(r=0.88)$. We found out the high correlation for Gait cycle time $(r=0.99)$, thus the system could constantly capture the human Gait from all trials. Considering works in literature [27, 31, 33], our system is capable of outputting spatiotemporal variables with higher correlation between Gait system and VICON. The literature on [25], an impressive work on Multiple Kinects, which has not compared their results with a gold standard system, reported results of 0.97 for right stride length, 0.83 for left stride length, and 0.92 for Gait time. Our results have shown correlation of 0.99 for Gait time and 0.95 for right and left stride length, which was a positive result. 
TABLE II

TECHNOLOGIES COMPARISON

\begin{tabular}{lcccc}
\hline \hline Feature & VICON & Multi-Kinect & IMU & $\begin{array}{c}\text { Multimodal } \\
\text { Gait System }\end{array}$ \\
\hline Marker-less & $x$ & $\checkmark$ & $\checkmark$ & $\checkmark$ \\
3D & $\checkmark$ & $\checkmark$ & $x$ & $\checkmark$ \\
$360^{\circ}$ View & $\checkmark$ & $\checkmark$ & $x$ & $\checkmark$ \\
Quick set-up & $x$ & $\checkmark$ & $\checkmark$ & $\checkmark$ \\
Low-cost & $x$ & $\checkmark$ & $\checkmark$ & $\checkmark$ \\
Body frame $^{\mathrm{a}}$ & $\checkmark$ & $\checkmark$ & $x$ & $\checkmark$ \\
Temporal Gait & $\checkmark$ & $\checkmark$ & $\checkmark$ & $\checkmark$ \\
Spatial Gait & $\checkmark$ & $\checkmark$ & $x$ & $\checkmark$ \\
Joint angles & $\checkmark$ & $x$ & $\checkmark$ & $\checkmark$ \\
\hline \hline
\end{tabular}

This table illustrates possible Gait features of VICON, Multi-Kinect, IMU, and the Gait System.

${ }^{a}$ Body frame refers to the capability of the system to reconstruct body with 3dimensional points.

\section{CONCLUSION}

In this work, we have presented a low-cost GAIT analysis system based on the integration of a Multi Kinect system and Shimmer inertial sensors. The novel multimodal system fuses IMU sensors (acceleration, gyroscope, and magnetometer) to get quaternion orientation and combines IMU data with 3D points of Multiple Microsoft Kinects (which employ RGB-D cameras and skeleton data) and output spatiotemporal Gait variables. It also includes a novel synchronization protocol for integration and synchronization of the multimodal modules in the entire system.

A summary of capabilities of our Gait system with other techniques described in this paper is shown in Table II. MoCap Technologies such as Vicon have high levels of precision and accuracy, however, difficulties with set up, high cost, and the use of markers provided barriers for use in applications and fields. Our system considers the use of IMUs as a form to enhance joint angles, it is marker-less which makes set up quick and easy, and provided accurate spatiotemporal Gait variables. Applications of the multimodal Gait system are beyond low-cost Gait analysis, it includes rehabilitation feedback, and Virtual and Augmented Reality applications which are topics of our future works.

The system performance was compared with the gold standard VICON system. Table II informs that the VICON system is capable to promote 3D information and can provide all Gait variables, however it still uses markers to capture 3D points and it is not low-cost. Our system price is around 5.000 EUR, while gold standard system is around 150.000 EUR. Both modules of the proposed Gait system can contribute to have a marker-less system, complete 3D and $360^{\circ}$, and can provide Gait spatiotemporal variables.

In summary, this paper presents analysis and discussion on signals differences between our proposed system and VICON; as well as angle estimation from inertial sensors integration. Our analysis has demonstrated the utility of our multimodal system (inclusive of its limitations). Based on this, many potential use cases of the Gait system can be proposed. The proposed system is cheaper; is easy to set up; shows clear and easily interpretable results; is marker-less; supports 360 degrees of motion analysis; is easily portable and does not require large set up space or environment. Analysis and consideration of the results infers that our system was capable of replicating Gait angles of Knee and Hip with 95\% bootstrap confidence interval. Spatiotemporal results showed similarity with significant results of $99 \%$ on Gait cycle time (s), $95 \%$ on right stride time (s), $90 \%$ on left stride time (s), $88 \%$ on Gait length $(\mathrm{m}), 95 \%$ on stride length $(\mathrm{m})$, and $91 \%$ on Gait velocity $(\mathrm{m} / \mathrm{s})$.Future work of this project will include the use of Gait analysis system the use it in real-time as an immersive multimedia haptic/Augmented Reality feedback tool for Gait reeducation.

\section{ACKNOWLEDGMENT}

The work presented in this paper has been supported by the Irish Research Council under grant GOIPG/2017/803. This publication has emanated from research conducted with the financial sup- port of Science Foundation Ireland (SFI) under grant number $\mathrm{SFI} / 12 / \mathrm{RC} / 2289$.

\section{REFERENCES}

1. Ferreira, A.V.S., et al., Comparison of motor function in patients with Duchenne muscular dystrophy in physical therapy in and out of water: 2-year follow-up. Acta Fisiatrica, 2015. 22(2): p. 51-54.

2. Shivesh, K., Contribution to modeling of human walking gait cycle over stride based on robotics for pedestrian navigation solution. 2015, Ecole Centrale de Nantes: Nantes. 

2006. 2006. Southampton.

Derlatka, M. Human Gait Recognition Based on Signals from Two Force Plates. in International Conference on Artificial Intelligence and Soft Computing. 2012. Berlin.

5. Lei, J., et al. Gait analysis of human locomotion based on motion capture system. in 2014 IEEE 9th Conference on Industrial Electronics and Applications (ICIEA). 2014. Hangzhou.

Mündermann, L., S. Corazza, and T.P. Andriacchi, The evolution of methods for the capture of human movement. Journal of NeuroEngineering and, 2006. 3(6).

Ceseracciu, E., Z. Sawacha, and C. Cobelli, Comparison of Markerless and Marker-Based Motion Capture Technologies through Simultaneous Data Collection during Gait: Proof of Concept. PLoS One, 2014. 9(3).

Vicon. Vicon Motion Systems Ltd.

Windolj, M., N. Gotzen, and M. Morlock, Systematic accuracy and precision analysis of video motion capturing systems--exemplified on the Vicon460 system. Journal Biomechanics, 2008. 41(12): p. 2276-2280.

Tarnita, D., Wearable sensors used for human gait analysis. Romanian Journal of Morphology \& Embryology, 2016. 57(2): p. $373-382$.

Chen, P.-z., et al., Real-Time Human Motion Capture Driven by a Wireless Sensor Network. International Journal of Computer Games Technology, 2015. 2015: p. 1-15.

Andriacchi, T.P. and E.J. Alexander, Studies of human locomotion: past, present and future. Journal of Biomechanics, 2000. 33(10): p. $1217-1224$.

Patrizi, A., E. Pennestri, and P.P. Valentini, Comparison between low-cost marker-less and high-end marker-based motion capture systems for the computer-aided assessment of working ergonomics. Vol. 59. 2015. 1-11.

Sellers, W.I. and E. Hirasaki, Markerless 3D motion capture for animal locomotion studies. Biology Open, 2014. 3: p. 356-368.

Microsoft, Kinect for Xbox One. 2015, Microsoft Corporation.

Jana, A., Understanding the Kinect Device, in Kinect for Windows SDK Programming Guide. 2014, Packt Publishing. p. 392.

Gutub, A., Exploratory Data Visualization for Smart Systems. 2015.

Alharthi, N. and A. Gutub, Data Visualization to Explore Improving Decision-Making within Hajj Services. Vol. 2. 2017. 9-18.

Abdelgawad, H., et al., Microscopic modeling of large-scale pedestrian-vehicle conflicts in the city of Madinah, Saudi Arabia. Vol. 48.2014.

Kaysi, I., et al., Users' Evaluation of Rail Systems in Mass Events: Case Study in Mecca, Saudi Arabia. Vol. 2350. 2013. 111-118.

Gutub, A. and N. Alharthi, Improving Hajj and Umrah Services Utilizing Exploratory Data Visualization Techniques. 2016.

Gutub, A., et al., Velocity-Based Modeling of Physical Interactions in Dense Crowds. Vol. 31. 2014.

Eichelberger, P., et al., Analysis of accuracy in optical motion capture - A protocol for laboratory setup evaluation. Journal of Biomehanics, 2016. 49: p. 2085-2088.

B Rodrigues, T., et al. An Evaluation of a 3D Multimodal Marker-less Motion Analysis System. in ACM Multimedia Systems Conference 2019. 2019. Amherst, MA, USA.

Castelli, A., et al., A 2D Markerless Gait Analysis Methodology: Validation on Healthy Subjects. Computational and Mathematical Methods in Medicine, 2015. 2015(186780).

Saboune, J. and F. Charpillet, Markerless Human Motion Tracking from a Single Camera Using Interval Particle Filtering. International Journal on Artificial Intelligence Tools, 2007. 16(4): p. 593-609.

Gabel, M., et al. Full Body Gait Analysis with Kinect. in 34th Annual International Conference of the IEEE EMBS. 2012. San Diego.

Clark, R.A., et al., Concurrent validity of the Microsoft Kinect for assessment of spatiotemporal gait variables. Journal of Biomechanics, 2013. 46: p. $2722-2725$.

Systems, S., Clincal 3DMA. Stt Systems.

Choppin, S., B. Lane, and J. Wheat, The accuracy of the Microsoft Kinect in joint angle measurement. Sports Technology, 2014. 7(1-2): p. 98-105.

Muller, B., et al., Validation of enhanced kinect sensor based motion capturing for gait assessment. PLoS ONE, 2017. 12(4): p. 1-18.

V, K., Preface, in Inertial MEMS: Principles and Practice. 2011, Cambridge University Press: Cambridge. p. Xiii-Xiv.

Henri, D.V., et al., The implementation of inertial sensors for the assessment of temporal parameters of gait in the knee arthroplasty population. Clinical Biomechanics, 2018. 54: p. 22-27.

Ferrari, A., et al., A Mobile Kalman-Filter Based Solution for the Real-Time Estimation of Spatio-Temporal Gait Parameters. IEEE Transactions on Neural Systems and Rehabilitation Engineering, 2016. 24(7): p. 764-773.

The GAITRite standard.

Sebastian O. H., M., H. Andrew J. L., and V. Ravi. Estimation of IMU and MARG orientation using a gradient descent algorithm. in 2011 IEEE International Conference on Rehabilitation Robotics. 2011. Zurich.

Microsoft, Kinect for Windows SDK 2.0. () Microsoft.

Berthold K. P., H., Closed-form solution of absolute orientation using unit quaternions. Journal of the Optical Society of America, 1987. 4(4): p. 629642.

Shimmer, Shimmer3 IMU Unit. (@ Shimmer.

Howell, A.M., et al., Kinetic Gait Analysis Using a Low-Cost Insole. IEEE Transactions on Biomedical Engineering, 2013. 60(12): p. $3284-3290$.

Karg, M., K. Kühnlenz, and M. Buss, Recognition of Affect Based on Gait Patterns. IEEE Transactions on Systems, Man, and Cybernetics, 2010. 40(4): p. $1050-1061$.

Clark, R.A. and A.L. Bryant, Validity of the Microsoft Kinect for assessment of postural control. Gait \& Posture, 2012. 36(3): p. $372-377$.

Plug-in Gait Reference Guide. 2018, Vicon Motion Systems.

Fill gaps in trial data. 2018, Vicon Motion Systems.

Lau, E., et al., Factors Associated with Osteoarthritis of the Hip and Knee in Hong Kong Chinese: Obesity, Joint Injury, and Occupational Activities. American Journal of Epidemiology, 2000. 152(9): p. 855-862.

Calce, S.E., et al., The relationship of age, activity, and body size on osteoarthritis in weight-bearing skeletal regions. International Journal of Paleopathology, 2018. 22: p. 45-53.

Kujala, U., J. Kaprio, and S. Sarna, Osteoarthritis of weight bearing joints of lower limbs in former elite male athletes. BMJ: British Medical Journal, 1994. 308(6923): p. 231-234.

Kale et al., A., Identification of humans using gait. EEE Transactions on Image Processing, 2004. 13(9): p. 1163-1173.

Muro-de-la-Herran, A., B. Garcia-Zapirain, and A. Mendez-Zorrilla, Gait Analysis Methods: An Overview of Wearable and Non-Wearable Systems, Highlighting Clinical Applications. Sensors, 2014. 14(2): p. 3362-3394.

Bacon-Shone, V.C. and J. Bacon-Shone, Gait of Normal Hong Kong Chinese Children: The Bootstrap Approach. Hong Kong Physiotherapy Journal, 2000. 18(1): p. 21-25.

Lenhoff, M.W., et al., Bootstrap prediction and confidence bands: a superior statistical method for analysis of gait data. Gait and Posture, 1999. 9: p. $10-17$. 


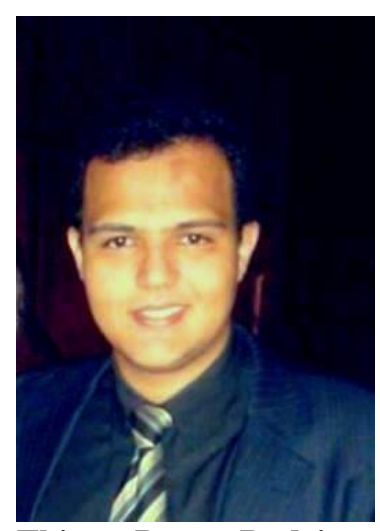

Thiago Braga Rodrigues is a research candidate in the Athlone Institute of Technology (AIT), Ireland. He has a BEng in Biomedical Engineering from the Universidade Federal de Uberlândia in 2015 and is currently in pursuit of his PhD degree in Software Engineering. His current research work includes the use of wearable sensor systems on evaluating the utility and relationship of different feedback mechanisms in Gait analysis using various physiological metrics and user quality of experience.

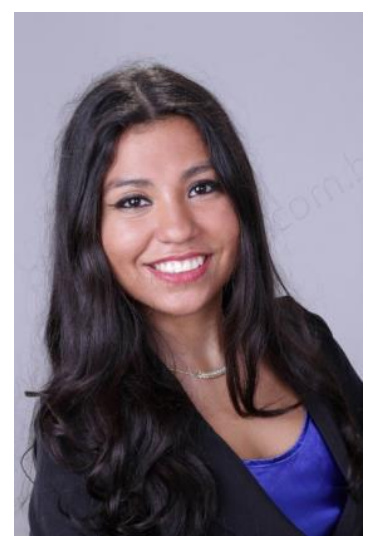

Débora Pereira Salgado is a research candidate in the Athlone Institute of Technology (AIT), Ireland. She has a BS. in Biomedical Engineering from the Universidade Federal de Uberlândia in 2017 and is currently in pursuit of her PhD. Her current research work focuses on evaluating the utility and relationship of various physiological metrics and user quality of experience of emerging immersive multimedia experiences.

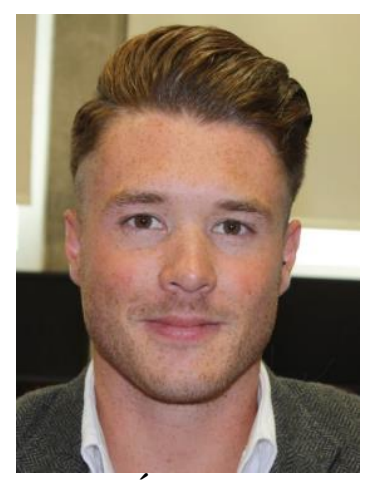

Ciarán Ó Catháin is a lecturer in Sport Science in Athlone Institute of Technology. Dr Ó Catháin's research interests focuses on multi-disciplinary approaches to improving sporting performance and reducing injury risk. This includes working closely with researchers across multiple disciplines such as biomechanics, exercise physiology, nutrition, and software engineering to provide a more holistic understanding of the underlying mechanisms that can be targeted to improving athletic performance and reduce injury development. In particular, Ciarán has focused on the development of novel technologies that provide real-time biofeedback to runners with the goal of reducing the risk of injury development. 


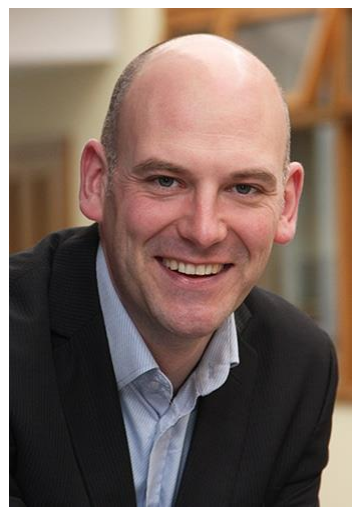

Noel E. O'Connor is a Professor in the School of Electronic Engineering at DCU and the Academic Director of the Research and Enterprise Hub on Information Technology and the Digital Society. He is a Funded Investigator (FI) in Insight, Ireland's national Centre for Data Analytics funded by Science Foundation Ireland. Since 1999 Prof. O'Connor has published over 180 peer-reviewed publications, made 11 standards submissions and filed 6 patents. He has graduated 16 Ph.D students and 3 Masters students. Noel's early research was in the field of video compression, specifically object-based compression in the context of MPEG-4, which subsequently led to an interest in video object segmentation and tracking as well as other aspects of computer vision. With the advent of MPEG-7, he became interested in audio-visual (AV) analysis for content-based information retrieval as well as lowpower configurable hardware for AV processing, a key-enabler for next generation context-aware multimedia sensors.

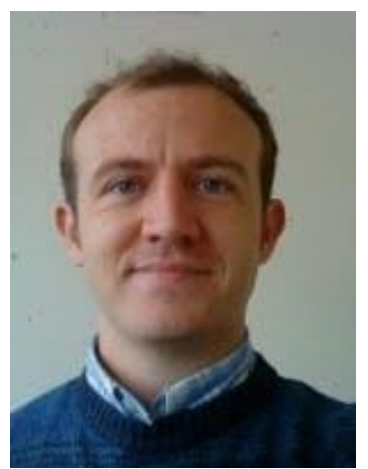

Niall Murray is a Lecturer with the Faculty of Engineering and Informatics, in the Athlone Institute of Technology (AIT), Ireland. $\mathrm{He}$ is founder (in 2014) and principal investigator (PI) in the truly Immersive and Interactive Multimedia Experiences (tIIMEx) research group in AIT. He is a Science Foundation Ireland (SFI) Funded Investigator (FI) in the Confirm Centre for Smart manufacturing and an associate PI on the Enterprise Ireland funded Technology Gateway COMAND. His current research interests include immersive and multisensory multimedia communication and applications, multimedia signal processing, quality of experience, and wearable sensor systems. He has published over 40 works in top-level international journals and conferences and book chapters. Further information available at: www.niallmurray.info 